\title{
IDENTITIES IN FINITE ALGEBRAS
}

R. C. LYNDON

A finite algebraic system $\mathcal{A}$ will be constructed, with the property that the set of all laws of $\mathcal{A}$ is not a consequence of any finite subset of these laws. ${ }^{1}$

$A$ is of order seven, with a binary (non-associative) operation written as multiplication, and a 0 -ary operation (or constant element) 0 . Denoting the elements of $A$ by $0, e, b_{1}, b_{2}, c, d_{1}, d_{2}$, the multiplication is defined by setting

$$
c e=c, \quad c b_{j}=d_{j}, \quad d_{j} e=d_{j}, \quad d_{j} b_{k}=d_{j} \quad(j, k=1,2)
$$

and setting all other products equal to 0 .

Lemma 1. The following laws hold in $A$ :

$$
0 x=0, \quad x 0=0, \quad x(y z)=0 ;
$$

$(\mathrm{B} n)$ :

$$
\left(\left(\cdots\left(\left(x_{1} x_{2}\right) x_{3}\right) \cdots\right) x_{n}\right) x_{1}=0 \quad(n=1,2, \cdots) ;
$$

$(\mathrm{C} n)$ :

$$
\begin{array}{r}
\left(\left(\cdots\left(\left(x_{1} x_{2}\right) x_{3}\right) \cdots\right) x_{n}\right) x_{2}=\left(\cdots\left(\left(x_{1} x_{2}\right) x_{3}\right) \cdots\right) x_{n} \\
(n=1,2, \cdots) .
\end{array}
$$

Moreover, every law of $A$ that contains no more than $n$ indeterminates is a consequence of $\mathrm{A} 123$ and of $\mathrm{B} m, \mathrm{C} m$ for $m \leqq n$.

Proof. Verification that $\mathrm{A} 123, \mathrm{~B} n$ and $\mathrm{C} n$ hold identically in $\mathcal{A}$ is routine. For the second assertion, let $\phi_{\alpha}=\phi_{\beta}$ be any law of $A$ containing at most $n$ indeterminates. By $\mathrm{A}$ we may suppose that each member is either 0 or a left-associated product, which we abbreviate as

$$
\phi_{\alpha}=x_{\alpha_{1}} x_{\alpha_{2}} \cdots x_{\alpha_{\mu}}, \quad \phi_{\beta}=x_{\beta_{1}} x_{\beta_{2}} \cdots x_{\beta_{\nu}} .
$$

By $\mathrm{B} m$ and $\mathrm{C} m$ for $m \leqq n$, we can eliminate any repetition among the first $n+1$ arguments of $\phi_{\alpha}$ or of $\phi_{\beta}$, whence we may suppose that $\mu, \nu \leqq n$ and that neither product contains repetitions. If $\phi_{\alpha}$, say, is not 0 , then setting $x_{\alpha_{1}}=c, x_{\alpha_{2}}=\cdots=x_{\alpha_{\mu}}=e$, and any other $x=0$, gives $\phi_{\alpha}=c$; it follows that $\phi_{\beta}$ is not 0 , and indeed that $x_{\beta_{1}}=x_{\alpha_{1}}$, and that all the indeterminates of $\phi_{\beta}$ are among those of $\phi_{\alpha}$. By sym-

Presented to the Society, February 28, 1953; received by the editors May 2, 1953.

1 The author is indebted to J. Kalicki for a simplification of the algebra A. For general reference see Trans. Amer. Math. Soc. vol. 71, p. 457 (bibliography), also Proc. Amer. Math. Soc. vol. 3, p. 579, and J. Kalicki, Proc. Amer. Math. Soc. vol. 3, p. 36. 
metry, $\mu=\nu$ and the $x_{\alpha_{i}}$ are a permutation of the $x_{\beta_{i}}$. If some $x_{1}$ preceded $x_{2}$ in $\phi_{\alpha}$, while $x_{2}$ preceded $x_{1}$ in $\phi_{\beta}$ (therefore $x_{\alpha_{1}} \neq x_{1}, x_{2}$ ), then setting $x_{\alpha_{1}}=c, x_{1}=b_{1}, x_{2}=b_{2}$ and all other $x=e$ gives $\phi_{\alpha}=d_{1}$ and $\phi_{\beta}=d_{2}$. Since this contradicts $\phi_{\alpha}=\phi_{\beta}$, the $x_{\beta_{i}}$ must be the identical permutation of the $x_{\alpha_{i}}$, whence $\phi_{\alpha}$ and $\phi_{\beta}$ are formally identical.

Define $\psi_{\alpha}=x_{\alpha_{1}} x_{\alpha_{2}} \cdots x_{\alpha_{\mu}}$ to have property $\mathrm{P} n$ if $\psi_{\alpha}$ contains $n$ indeterminates, and in the product $\psi_{\alpha}$ each of them occurs at least once to the left of the second occurrence of $x_{\alpha_{1}}$. Note that this implies that if $x_{\alpha_{k}}$ is a later occurrence of $x_{\alpha_{1}}$, then $k>n$.

LEMMA 2. If $\psi_{\alpha}=\psi_{\beta}$ is a formal consequence of the laws $\mathrm{A}$ and $\mathrm{B} m$, $\mathrm{C} m$ for $m<n$, and if $\psi_{\alpha}$ has the property $\mathrm{P} n$, then $\psi_{\beta}$ also has the property $\mathrm{P}$.

Proof. It is sufficient to consider the case where $\psi_{\alpha}=\psi_{\beta}$ results from a single application of $\mathrm{A}, \mathrm{B} m$, or $\mathrm{C} m$. Now, no application of $\mathrm{A}$ is possible, since $\psi_{\alpha}$ contains no part 0 or of the form $u(v w)$. For an application of $\mathrm{Bm}$ or $\mathrm{Cm}$ we may suppose, by symmetry, that $\psi_{\alpha}$ contains a part of the form $y_{1} y_{2} \cdots y_{m} y_{d}$, where $y_{d}=y_{1}$ in the case of $\mathrm{B} m$ and $y_{d}=y_{2}$ in the case of $\mathrm{C} m$. Clearly $y_{1}=x_{\alpha_{1}} \cdots x_{\alpha_{k}}$ for some $k \geqq 1$, and therefore $y_{2}=x_{\alpha_{k+1}}, \cdots, y_{d}=x_{\alpha_{k+m}}$. For $\mathrm{B} m, y_{d}=y_{1}$ $=x_{\alpha_{1}} \cdots x_{\alpha_{k}}$ is clearly impossible for $k>1$; while if $k=1, y_{d}=x_{\alpha_{m+1}}$ is a later occurrence of $y_{1}=x_{\alpha_{1}}$, impossible by property $\mathrm{P} n$ since $m+1$ $\leqq n$. Therefore no $\mathrm{B} m, m<n$, is applicable. Finally, if some $\mathrm{C} m$ is applicable it can only delete an occurrence after the first of some indeterminate other than $x_{\alpha_{1}}$, or else an occurrence after the second of $x_{\alpha_{1}}$, and hence in either case preserves the property $\mathrm{P} n$.

THEOREM. If $\mathrm{L}$ is any finite set of laws of $\mathcal{A}$, then some one of the laws $\mathrm{B} n$ is not a consequence of $\mathrm{L}$.

Proof. The finite set $\mathrm{L}$ of laws will contain fewer than $n$ indeterminates, for some finite $n$. By Lemma 1 , any consequence of $\mathrm{L}$ is a consequence of $\mathrm{A} 123$ and $\mathrm{B} m, \mathrm{C} m$ for $m<n$. Now $x_{1} x_{2} \cdots x_{n} x_{1}$, the left member of $B n$, has property $\mathrm{P} n$, while 0 , the right member, has not. Therefore, by Lemma $2, \mathrm{~B} n$ is not a consequence of $\mathrm{A} 123$ and $\mathrm{B} m, \mathrm{C} m$ for $m<n$, whence $\mathrm{B} n$ is not a consequence of $\mathrm{L}$.

Princeton University 